\title{
WILLIAM WHEWELL'S SEMANTIC ACCOUNT OF INDUCTION
}

\author{
Corey Dethier
}

\begin{abstract}
William Whewell's account of induction differs dramatically from the one familiar from twentieth-century debates. I argue that Whewell's induction can be usefully understood by comparing the difference between his views and more standard accounts to contemporary debates between semantic and syntactic views of theories: rather than understanding inductive inference as capturing a relationship between sentences or propositions, Whewell understands it as a method for constructing a model of the world. The difference between this ("semantic") view and the more familiar ("syntactic") picture of induction is reflected in other aspects of Whewell's philosophy of science, particularly his treatment of consilience and the order of discovery.
\end{abstract}

What is meant by success in these cases? To this we reply, that our inquiry must be, whether the facts have the same relation in the hypothesis which they have in reality. (William Whewell 1840/2014b, 210)

\section{Introduction}

To contemporary eyes, William Whewell's use of the term "induction" is idiosyncratic: it involves "superinducing" an "ideal conception" (sometimes, a "hypothesis") onto the empirical facts in order to connect them together (1840/ $2014 \mathrm{a}, 42)$. On Whewell's view, this process of combining the right active "idea" with passive "sensations" (26) is the method "by which the sciences now

Contact Corey Dethier at the Department of Philosophy, University of Notre Dame, 100 Malloy Hall, Notre Dame, IN 46556 (corey.dethier@gmail.com).

I would like to thank the audience at the 2017 Notre Dame History of Philosophy of Science Graduate Student Workshop, a number of anonymous reviewers, and especially Don Howard for insightful comments on earlier versions of this article.

HOPOS: The Journal of the International Society for the History of Philosophy of Science, vol. 8 (Spring 2018). 2152-5188/2018/0801-0007\$10.00. (C) 2018 by the International Society for the History of Philosophy of Science. All rights reserved. Electronically published February 9, 2018. 
existing among men have been constructed" (1849, 12-13). This atypical characterization of induction has inspired a variety of readings. Mill (1868/2012, 506), for instance, ascribes to Whewell the view that induction is merely the "trying of hypothesis after hypothesis until one is found which fits the phenomena," while Achinstein $(2010,729)$ sums up more recent commenters on Whewell as "a disparate group [of philosophers] who classify him as one of their own, as a hypothetico-deductivist, a defender of inference to the best explanation, a Quinean holist, or even a Kantian a priorist.”

As Snyder $(2008,182)$ has noted, however, one of the common themes of this literature has been a skepticism about just how inductive Whewell's "induction" really is. Thus, Buchdahl $(1991,313)$ characterizes Whewell's view as "very much in the spirit of the kind of methodology espoused in our time by the Popperian dynamic approach. . . . Any 'logical' movement that exists here is entirely from hypotheses to the data." Similarly, Butts $(1967,182)$ argues that in Whewell's writings, "induction" simply refers to the hypothetico-deductive method. Fisch (1991, 110) considers Whewell's "induction" to be essentially Peirce's "abduction" and approvingly quotes W. H. Davis's claim that, on this Peircean view, "scientific reasoning does not depend upon induction at all!" (109). And Achinstein $(1992,358)$ himself takes (or at least took) a similar interpretative position, arguing that Whewell's consilience is a form of inference to the best explanation rather than induction properly so called.

By contrast, I am convinced by Snyder's (2008, 219-20) argument that while Whewell does embrace certain elements of idealism in his broader "antithetical" epistemology, reading his inductive method as a form of hypothetico-deductivism or IBE (inference to the best explanation) would be a mistake. ${ }^{1}$ The confusion stems, Synder argues, from an overly restrictive understanding of induction owed to twentieth-century debates between Millian inductivists and hypotheticodeductivists, debates that make it seem as though the Millian picture of induction is the only possible one (221). I think Snyder is correct: Whewell does have a markedly different view of induction than that normally found in contemporary accounts, and better understanding the contrast between Whewell's view and a standard twentieth-century picture can help us better understand not only this vision of induction but Whewell's epistemology more broadly. In this essay, I develop the contrast more explicitly using a comparison to debates over the nature of theories: Whewell differs from twentieth-century views on induction in

1. The issue here is not whether Whewell recognizes hypothetico-deductive reasoning or inference to the best explanation as legitimate but whether Whewell understands induction in a manner that we might identify with these methods. My thanks to an anonymous reviewer for pressing me on this point. 
much the same way that the semanticist differs from the defender of syntactic views.

I begin by briefly laying out Whewell's general epistemology of science. Then, bringing together recent work by Snyder (2005) and Forster (2011), I argue that on Whewell's account, induction should be understood as an ampliative model-building procedure that is successful if the abstract objects that it creates have "the same" structure as found in the world. ${ }^{2}$ As such, this procedure is usefully seen as akin to the semantic view of theories in the way that it presents an alternative to the more sentence- or proposition-focused accounts of induction prevalent in twentieth-century debates. Not only does this comparison help us make sense of Whewell's explicit comments on induction; it also illuminates other aspects of his philosophy of science. In the final section, I turn to two well-known characteristics of his epistemology of science-the value of consilience and the justificatory importance of the order of discovery-to argue that both align with this "semantic" account of induction in a way that they do not with the contrasting "syntactic" picture.

Before beginning, however, a brief note. Whewell was clearly concerned with the nature of theories and the proper understanding of their representative character (see, e.g., Whewell 1840/2014a, xli) and equally if not more concerned with how we justify these representations (see, e.g., Whewell 1840/2014a, xxxix$\mathrm{xl})$. He shared these concerns with predecessors such as Thomas Reid (1785/ 1941, 33, 58) and Kant (1786/2004, 4-6), opponents such as Mill (1868/ 2012, bk. 3), and philosophically inclined scientists such as John Herschel $(1830 / 1966,164-70)$ and James Clerk Maxwell, famous for his use of alternative means to represent physical phenomena. ${ }^{3}$ The framing of these questions in terms of a debate between syntactic and semantic accounts of theories is a twentieth-century phenomenon, however, and it would be a mistake to assimilate these authors to it, something that I have no intention of doing. My use of terms like "semantic" and "syntactic" in this essay is meant to help us understand, by way of comparison, the difference between Whewell's unfamiliar views on induction and more familiar ones; it is similar to the difference between semantic and syntactic accounts of theories. As we will see, the comparison is fruitful, but it pays to remember that Whewell and his contemporaries understood the relevant issues within a framework dramatically different from our own.

2. Of course, I do not think that Whewell has in mind anything like the truth-making function of mathematical models sometimes associated with the semantic view of theories. See Thomson-Jones (2006) for discussion of the different senses of "model" at use in semantic views.

3. For the influence of Kant and Scottish common sense philosophy on Whewell, see Snyder (2006, esp. 46). For Whewell's influence on Maxwell, see Harman (1998, esp. 32-36). 


\section{Whewell's Philosophy of Science}

For Whewell (1840/2014a, 3), a finished philosophy of science would offer "a complete insight into the essence and conditions of all real knowledge, and an exposition of the best methods for the discovery of new truths." The first part of this project is determining the nature of scientific knowledge. On his account, any and all knowledge of the empirical world involves both passive and active capacities of the mind (26). Passively, the mind takes in sensations produced by the external world. These sensations are not merely received, however, and Whewell is clear that there cannot be any sort of knowledge on the basis of sensation alone. Even "recogniz[ing] any single body as existing" (26) requires actively "imposing" ideas on the sensations that are given to us. The ideas imposed on our sensations reflect the conditions of the world, not internal facts about our minds. As Snyder $(2008,170)$ puts it, they "provide the general relations that really exist in the world between objects and events."

If knowledge is the combination of ideas and sensations, induction-the "best method" (Whewell 1840/2014a, 4) for reaching knowledge-is the act of combining ideas with sensations. On Whewell's account, this act is broken into two major inseparable "operations" of the sciences, the explication of ideas and the colligation of facts $(1840 / 2014 \mathrm{~b}, 212) .{ }^{4}$ The first of these two operations involves the extension and clarification of ideas, the second the application of an idea to the world such that the facts are properly united in the resulting mental construction. It is important that these two processes are inseparable: at least in practice, fully developing all of our ideas before attempting to apply them to the world is impossible. Instead, explication seems to be largely a matter of repeated attempts to unite the facts under an idea (225). For Whewell, an explicit definition of an idea is a useful if unnecessary end to the process of explication (179), and such definitions are typically the product of a successful colligation (219) — we usually come to a proper definition of an idea only through wrestling with the empirical facts that we are using it to unify.

Given that Whewell views knowledge as the successful combination of ideas with sensations, it is no surprise that he understands induction primarily as a process that produces this type of combination of passive and active elements. More idiosyncratic than his understanding of the results of this process is Whe-

4. Near the beginning of vol. 1 of The Philosophy of the Inductive Sciences, Whewell (1840/2014a, xliii) lists three "steps" rather than the two "operations": "the Selection of Idea, the Construction of the Conception, and the Determination of the Magnitudes." I do not believe that these two characterizations conflict. As I read him, the description in terms of operations is a more abstract account of the justificatory process, while the three "steps" describe temporal stages of that process. Regardless, the two operations have a much larger role in The Philosophy of the Inductive Sciences. 
well's understanding of the process itself. One familiar method for introducing students to induction is via schemas of the form "All observed $x$ 's have property $P$; therefore, all $x$ 's have $P$ " or "All observed $x$ 's have property $P$; therefore, the next $x$ will have property $P$." At least superficially, what makes these examples inductive is the move from observed instances to the further conclusion. For Whewell $(1840 / 2014 \mathrm{a}, 66)$, however, the proper form of the induction is better expressed in terms of the unification of the idea of $x$ and the idea of $P$ into a general proposition $(P x)$ and the use of this new idea to characterize the observed facts. Notice that while this vision of induction does involve the unification of particular facts (Whewell 1840/2014a, xl), it does not involve an inference from a claim about particulars to a general fact. Instead, the general fact is constituted by the impression of the idea on the particulars $(1840 / 2014 \mathrm{~b}$, 213). As such, Whewell's induction differs dramatically from familiar accounts of deductive or syllogistic inferences: an inductive inference is not just deductive inference where entailment fails; it is something altogether different.

\section{Whewell's Semantic Account of Induction}

In order to bring out the unique aspects of Whewell's account of induction, it will be helpful to have on hand an opposing account in which inductive and deductive reasoning are more closely related - that is, one in which an inductive inference is like a deductive inference but ampliative or probabilistic (or both) rather than deductively valid. For our purposes, Carnap (1962) provides a useful foil..$^{5}$ There are three central theses of his view that I want to focus on:

(1) Both the premises and conclusions of an inductive inference are propositional or sentential.

(2) Inductive reasoning (in the sense relevant to the epistemology of science) is characterized by failure of the premises to entail the conclusion.

(3) "Strength," the inductive analogue of validity, is a function of the logical relation between the premises and the conclusion.

While each of these theses is drawn directly from Carnap (specifically Carnap 1962 , v), I take it that they are relatively widely endorsed or at least seen as a

5. In all but one respect: Carnap uses the term "semantic" in the logical sense of involving an interpreted formalism, and his account of both deduction and induction is semantic in this sense. By contrast, we are using "semantic" in the sense of more recent debates in philosophy of science: i.e., an account is semantic if it gives pride of place to abstract models rather than linguistic statements. It might therefore be preferable to term Whewell's view "model-centric" and foil "deductivist," but these terms have their own burdens and lack the clear connection to the present literature that I find illuminating. 
part of an intuitive package from which alternative accounts (such as Harman 1965, Kyburg 1990, or Norton 2003) depart. ${ }^{6}$ I will call the family of accounts exemplified by Carnap the "syntactic" view of induction owing to the emphasis that the three theses place on formal linguistic structures. As we will see, Whewell rejects all three, and I will argue that he does so in a way that is usefully understood by comparison with the semantic theorist's rejection of the syntactic accounts of theories.

Starting with thesis 1 , we have already seen that the elements of Whewell's induction are distinctly not sentences or propositions: the "premises" always include both facts and an idea that is then superimposed upon them (1840/ 2014a, 26). What are these components? For Whewell, a fact is an abstract object composed of sensations (or other facts) and an idea such that the idea gives form to the matter that is the sensations (31). As noted earlier, it is impossible to be given a sensation without its form, and Whewell thinks that the sole distinction between what we call a "fact" and what we call a "theory" is that we are aware that we have impressed an idea in the latter case (24). ${ }^{7}$ In other words, induction takes in some facts—roughly, sensations that are given form by ideasand unifies them under a new idea, showing that they share a common form. Or, to put it in terms Whewell would not use, an induction takes in abstract objects that are structured in certain ways and constructs a new, unifying abstract object by imposing additional structure.

The result of this process is a new fact, and Whewell $(1840 / 2014 \mathrm{~b}, 181)$ is not willing to guarantee that this abstract object will be expressible in language available to the scientist. Instead, he seems to understand it as a mental construction, and an induction is successful if the facts are united in "the same" manner in our construction as they are in the world (210). The criteria for sameness are underdeveloped in Whewell's account. It is clearly not isomorphism, but the exact metaphysical relationship is not spelled out in detail anywhere that I am aware of. Nevertheless, he does seem to have in mind something similar to the view defended by semantic accounts of theories: our theorizing is successful when we mentally combine (the right) facts in "the same" relationship that these

6. As one reviewer rightly stressed, it is not clear that this combination of principles retains this special status. Given the increased popularity of accounts that reduce induction to IBE along the lines suggested by Gilbert Harman, in particular, it is likely that the package is less prevalent today than it was a few decades ago.

7. While I will use both "theory" and "fact" in largely standard ways throughout the essay, this equivalence should be remembered. Where I intend to indicate specifically that a fact/theory unifies other facts (rather than sensations), I will contrast the unifying "higher-level theory" to the unified "lowerlevel fact," but these phrases should be understood as contextual to a particular inductive unification. 
facts have in the world. In the contemporary terms appealed to in the last paragraph, a successful induction creates a model of what really exists in the world rather than a true sentence. For Whewell, therefore, none of the elements of an induction are propositional: induction combines sensations with ideas like curve or ellipse to produce a mental representation — a fact or theory-of the world that may or may not be exactly expressed in the available language. ${ }^{8}$

An example is helpful, and Whewell provides one in the form of "inductive tables" in volume 2 of The Philosophy of the Inductive Sciences. ${ }^{9}$ On the broadest level, these tables proceed from phenomena (e.g., "The Tides ebb and flow") to theory ("All bodies attract each other with a Force of Gravity which is inversely as the square of the distances"), just as one would expect, given Whewell's explicitly inductive methodological viewpoint. More interesting are the elements between the predictable extremes, where Whewell includes inferences such as "Fall of heavy bodies [implies that] Earth attracts Moon invers. as square of distance (Newton)" and "Two Rhombs of Calcspar make 4 images alternately appear and disappear [implies that] Rays of light have four Sides with regard to which their properties alternate (Huyghens)." ${ }^{10}$ Obviously, these inferences are not intended to be simple relationships between sentences or propositions. On my interpretation, the premises (which are sometimes simply "By mechanics") give reasons to unify certain facts under a particular idea. Within a framework in which it has already been established that a force is directing the moon toward the earth, the "fall of heavy bodies" provides reason to model the motion of the moon according to an inverse-square attraction. Or, more in Whewell's terminology, the fall of heavy bodies reveals the fit between the fact of the motion of the moon and the idea of an inverse-square attraction, giving a good reason to impress this idea on the facts.

The second characteristic of the syntactic view of induction (thesis 2) is that induction is defined by its nondeductive nature. Given a focus on entailment between sentences or propositions, there are only two possible types of inference: those that involve entailment and those that do not. For Whewell, by contrast, induction is not best understood as an inference from one sentence or set of sentences to another. Instead, as we have seen, induction is better understood as a type of unification of various facts under a single idea to create a new fact. On this picture, the old facts are part of the new one in the sense of being constitu-

8. For Whewell, this is in contrast to deductive reasoning, which requires that the ideas be expressed in the form of axioms and definitions (1840/2014a, 89).

9. The tables occur between pp. 282 and 283 in my copy. They are also largely unreadable. Fortunately, Cambridge provides scans of the originals at cambridge.org/9781108064033 (under "Resources").

10. I have altered Whewell's presentation by substituting "[implies that]" for a horizontal line. 
ent pieces. In other words, the nature of induction is more modeling procedure than it is inference ticket: when we perform an induction, we create a new model out of a group of old models by bringing them together to constitute something new.

It needs to be stressed that Whewell's vision of induction is still ampliative in the sense that the new fact always goes beyond the observed facts that constitute it (1840/2014b, 214; cf. Snyder 2008). Whewell's own example illustrates the point adeptly. When Kepler superinduces an ellipse on Mars's orbit, Whewell regards his action as one of judging how to represent this orbit within a mathematical system in which the idea of an ellipse has certain necessary connections with other mathematical ideas. Generally, the ideas that we impose on facts already stand in certain relationships with other abstract objects - they are part of larger abstract structures. The idea imposed therefore unifies more than the observed facts; as Whewell $(1840 / 2014 a, 242)$ puts it, to impose an idea is to take it to be "universally valid." In our example, Kepler's unification of the positions of Mars's orbit under the idea of ellipse creates a structure that brings together not just the facts about observed positions but also facts about future positions. Whewellian induction is ampliative, therefore, because the model created when an idea is superinduced on facts always represents more of the world than has been observed.

The final characteristic of the syntactic view (thesis 3 ) is that strength is a function of the logical relations between the premises and the conclusion. Just as the validity of a syllogism is independent of its content, the strength of an induction is independent of whether we are generalizing about swans or ravens. The strength of a simple enumerative induction, for example, might be a function of the ratio between the size of the sample and the size of the population. Of course, content independence alone leaves quite a bit of room for disagreement about which properties characterize a strong induction. For Carnap (1962, v), strength ought to be a measurement of the probability of the conclusion relative to the probability of the premises. For Goodman (1979, 64), by contrast, a strong inductive schema is just one of a set of inductive rules that do not lead to contradictions.

For Whewell, neither of these conceptions of strength is even applicable to induction, as there is no one schema or general rule involved in multiple inductive inferences (1840/2014a, 66); nor, for that matter, does induction involve logical relations between sentences that can be characterized in this manner. Given that the essence of an induction is the application of a new idea to the facts - in contemporary terms, the creation of a new abstract object by the imposition of additional structure - the strength of an induction is primarily a function of the fit between the new idea applied and the facts. That is, it is pri- 
marily a function of whether the ideas are fit together in "the same" way in the idea as in the observed facts. Whewell's Kepler example is again helpful. What made Kepler's induction to elliptical orbits strong was not a relation between sentences but rather the "exact and universal accordance" between the idea of an ellipse and the observed facts (Whewell 1840/2014b, 169).

Whewell does not think that strength is only a function of fit, however, as he goes on to indicate in the sentence following the one just quoted. How well the imposition of the idea is supported by the rest of theory matters as well. A comparison with John Norton's "material induction" can help clarify this aspect of Whewell's thought. Like Whewell, Norton $(2003,650)$ denies that the inductive schema bears the burden of justifying a particular induction, relocating that justification to what he calls the "material postulate," which provides independent grounds for surety about the inference. Norton's material postulates are the results of prior science; to borrow one of his examples, we are much more secure in inductions relating to the melting temperature of elements than inferences regarding the color of fowl because we bring to these inductions knowledge about chemical kinds and animal pigmentation gained from prior inductions. The strength of the inductive inference thus depends on what we know about the kind that the induction is being applied to (cf. Snyder 2005, 130). While Whewell's ideas outrun Norton's explicit examples of material postulates rather dramatically (Snyder 2005, 131), there is a similarity: for Whewell, the strength of an induction depends on whether the abstract object produced can itself be unified to produce higher-level abstract objects, that is, whether we can show that elements in general have consistent melting temperatures. In the Kepler example, this effect is achieved when we show that Mars's elliptical orbit is not unique or accidental but is part of a larger pattern common to all planetary orbits. While an induction is judged first on the fit between the idea and factshow accurate the resulting model is - we can only really be sure of an inductive conclusion once its place in a whole system of supporting inductions has been identified (Whewell 1840/2014b, 169, 255; cf. Norton 2003, 668).

I have argued that Whewell rejects all three of the theses that I identified with the syntactic view of induction. As we have seen, he replaces them with the following:

(4) The knowledge resulting from a successful induction is a model that represents a part of the world by uniting the facts under the "same" relationships that they really stand in.

(5) An induction is defined by the ampliative imposition of an idea on the facts to create a new fact in which the old facts serve as constitutive parts. 
(6) The epistemic burden of justifying an inductive conclusion rests first on the fit between the specific idea and the facts and second on the fit of the conclusion in the broader model of related phenomena.

Given the nature of these three commitments, I think it is fair to call Whewell's view "semantic" owing to its likeness to the familiar position on the nature of theories: on my reading, he presents a view of induction that differs from more familiar accounts precisely in its focus on model building rather than logical relationships between sentences. Nevertheless, the method is still recognizable as induction. Although the ampliative and justificatory nature of induction is transformed by Whewell's approach, these characteristics remain central to his vision: the model generated by an induction goes beyond the facts and, if the induction is a good one, ought to be endorsed.

I think the picture presented above is accurate to Whewell's views, albeit presented in terms that he would not necessarily understand, let alone use. Still, the account should be understood as one that reflects my interests in this article: it leaves out significant aspects of his views, particularly relating to hypotheses, fundamental ideas, and God. There is much more to Whewell's philosophy of science than the views discussed above. In the rest of the article, I will show that the contrast between his semantic view and the more familiar syntactic one can help us understand and unify some of these other elements of Whewell's thought as well. In particular, I argue that two important characteristics of his epistemology - the value of consilience and the justificatory importance of the order of discovery-are better aligned with the semantic view of induction than they would be with the more familiar syntactic understanding.

\section{Semantic Induction and Whewell's Epistemology of Science}

\subsection{Consilience}

One of the interesting consequences of Whewell's $(1849,23)$ view that facts and theories are essentially the same is that any established theory can be used as a fact to be united under a new idea. The resulting layering of inductions is a nice instance of what Whewell has in mind in his discussions of consilience (see, e.g., Whewell 1840/2014b, 230; 1849, 61). Suppose we perform an induction on the observations of Mars by "superinducing" the concept of an ellipse onto its positions in the night sky. This process is itself a single induction that (if successful) establishes a true fact: the revolution of Mars is elliptical. Further separate inductions can be performed on the other planets, establishing that each has an elliptical orbit as well. These inductive inferences support each other- 
they are consilient - when we can make an additional induction that unifies them to show that planets in general revolve in ellipses (see Whewell 1840/ 2014b, 242-43). This instance of consilience, as Forster $(2011,94)$ stresses, is an example of bootstrapping in which the success of the lower-level induction is supported or confirmed by the higher-level one. In other words, the higherlevel theory can provide support to the lower-level induction by showing that the resulting facts fit into predictable and generalizable patterns.

Consilience based on different levels of facts is not the only type of consilience that Whewell recognizes. The fact that the same inverse-square gravitational relationship can be used to account for a variety of different phenomena is another example that he is particularly fond of (Whewell 1840/2014b, 230-31; see also Snyder 2005, 132-33). As Forster $(2011,105)$ notes, the uniting theme is the use of phenomena or aspects of phenomena that were not appealed to in establishing the original induction or inductions. In our example, the nature of Mars qua planet is not invoked in the original inference to elliptical orbits; this aspect is not employed as a reason for believing that its motion is elliptical. So, an induction that establishes that planets in general move in ellipses offers further evidence in support of our original induction by providing an additional reason to believe that Mars revolves in an ellipse-namely, the fact that it is a planet and that planets in general have elliptical revolutions.

On the syntactic view, consilience has no built-in role to play in an inductive inference, as the strength of an induction is simply a function of the logical relation between the evidence and the conclusion. ${ }^{11}$ Since consilience involves mutual support among a variety of different inductive inferences or conclusions, it is necessarily external to this relation. In our example, our inferences relating to each orbit are mutually supporting because in each case we find that the orbit is elliptical, but this does not alter the fit between the idea and the facts being unified. Here, it is essential to remember that induction, for Whewell, also involves the explication of ideas. That an idea fits a limited range of facts does not ensure that it is the right idea to impress on those facts. Finding that the same idea has purchase in a variety of applications, by contrast, indicates that the process of explication has gone correctly. As such, it provides independent, albeit indirect, support to the original inductive unification by indicating that the idea imposed was a good one, that is, that we successfully identified a genuine regularity or (what we might now call) a natural kind (cf. Snyder 2005, 138). Or: the fact that there is a common structure unifying the abstract models that we use to represent the world gives us indirect evidence that each one of these models is accurate by

11. Which is not to say that it is impossible for a sophisticated syntactic account to recover this role, as Myrvold (2003) attempts to do. 
providing us with evidence that our choice of model has not been influenced by arbitrary or parochial aspects of the particular phenomena being represented. Not only does consilience fit nicely with the semantic view's claim that inductive conclusions are further evidenced by being unified within higher-level or broader theories; it also helps explain why the defender of semantic induction would see this condition as important. If the emphasis is on the fit between the idea and the world, rather than the pure logical connections, the content of the idea (and thus getting the content of the idea right) is evidentially relevant. ${ }^{12}$

\subsection{The Justificatory Importance of the Order of Discovery}

The second aspect of Whewell's general epistemology of science that I wish to place emphasis on is his belief in what I will term the justificatory importance of the order of discovery, which played a central role in his famous disagreement with John Stuart Mill. In A System of Logic, Mill $(1868 / 2012,500)$ states that "if the supposition accords with the phenomena there needs no other evidence of it," seemingly endorsing the idea that the origins of a hypothesis are irrelevant when it comes to testing whether or not it is true (cf. Mill 1868/2012, 496). ${ }^{13}$ Whewell takes issue with this claim, but his response is obscure: he states that this view "is tainted with the vice ... of throwing the whole burthen of explanation on the unexplained word fact-unexplained in any permanent and definite opposition to theory" $(1849,62-63)$. What does the notion of "fact" have to do with whether or not it is valid to confirm a hypothesis by way of its predictions? Consilience-notably, the topic Whewell discusses on either side of this passage (see Whewell 1849, 61, 65)_offers a hint. As we noted above, the layering of inductions on top of each other can provide indirect support for the inductions on the lower level, and Whewell (1840/2014b, 244-45) is clear that the support provided by consilience is crucial to the verification of higher-level scientific theories such as Newtonian gravity. In effect, as Forster $(2011,104)$ again points out, the structure of induction on top of induction

12. For a more thorough discussion of Whewell's views on the development of ideas in the context of a specific scientific example, see Cobb (2011, particularly 88-89). My thanks to an anonymous reviewer for pointing out that the example employed in an earlier version of this paragraph was misleading.

13. I do not mean to imply that Mill held what I have been calling a syntactic view of induction. It is an interesting question whether the other major inductivists whom Whewell was familiar with-e.g., Bacon, Mill, Newton, or Reid (see Reid 1785/1941, 31) — held views accurately characterized by either side of the dichotomy that I have presented. Unfortunately, that question is outside the scope of this essay; my focus in this section is on arguing that Whewell's response to Mill can be understood as part of his broader semantic view on induction. For a more thorough examination of this debate and what is at stake within it, see Snyder (2008), Cobb (2011), or Forster (2011). 
gives the theory additional empirical predictions. For Whewell, Newtonian gravity unifies (and thus makes predictions about) more than observed positions. It also makes predictions about the characteristics of planets, comets, aphelia, and so on, as kinds, as Snyder (2005) argues. To simply hypothesize this theory and then test it ignores or at least obscures the structure of the theory and thus fails to independently test or confirm these crucial predictions (Snyder 2005, 135-36; cf. Whewell 1831, 399-400).

Whewell's main objection, therefore, is epistemological: he thinks that there is an important epistemic difference between testing a theory through his inductive method and "testing" a hypothesis merely by way of a comparison of its consequences with the facts. So, why does he say that the latter picture rests on a mistaken view of facts? Recall that Whewell thinks that there is no difference in kind between facts and theories_-both are the unification of sensations and ideas. Indeed, higher-level "theories" are constituted by inductions on lowerlevel facts: effectively, the former model includes the latter as parts that it unites together under a single idea. What Whewell objects to in Mill is what he reads as the denial of this constitutive relationship in favor of an opposition between theory and fact ("phenomena" in Mill's terms) in which one is tested against the other. Given the constitutive relationship between higher- and lower-level facts, by contrast, what actually needs to be tested are the inductions that bring the facts together at every level. Or, as Whewell (1840/2014b, 245) puts it, the only way to test a general theory is by "testing, at each step, both the reality of the asserted ingredients and the propriety of the conjunction." ${ }^{14}$ The structured view of justification just outlined is what I have in mind when I attribute to Whewell the view that the order of discovery has justificatory importance. The justification of a theory is necessarily piecemeal: it can only proceed through the confirmation of each induction, step by step. The obvious implication is that we cannot proceed by hypothesizing a higher-level theory and then confirming the whole of it by the comparison of its consequences with observed phenomena.

For Whewell, the nonpiecemeal procedure is unacceptable because it leaves the lower-level inductions untested: it does not check whether we have identified the correct ideas or merely developed a model that replicates the most superficial empirical features (Whewell 1831, 400). As was true with consilience, there is nothing about a syntactic induction that allows for his privileging of the

14. For Whewell, in other words, higher-level theories are confirmed in precisely the same way as lower-level facts are: we check whether the facts that are purported to be unified by the impression of the new idea are in fact unified. What differs is that a lower-level fact might unify only sensations, while a higher-level theory unifies many facts, which themselves may also unify facts. In order to properly test the higher-level theory, therefore, we need to ensure that the "facts" being unified are themselves veridical. My thanks to an anonymous reviewer for pressing me to clarify this issue. 
order of discovery. Indeed, on the syntactic account, it makes no difference whether the original inference to the inductive conclusion is confirmed or if some other consequence of the theory is. In both cases, the degree of confirmation is to be judged purely on the logical merits of the relation between sentences alone; syntactic inductions are insensitive to the order of discovery. On Whewell's semantic account, by contrast, insensitivity is impossible. Whewell rejects Mill's position because he thinks that theories are constituted by facts rather than tested against them. In contemporary terms, facts and theories are abstract objects, and higher-level theories such as Newtonian mechanics are just abstract objects that have other abstract objects as parts. While a theory can thus gain indirect support through the testing of any of its implications, it can only be properly confirmed by examining whether the facts that serve as its parts are both veridical and properly unified (Whewell 1840/2014b, 245). Since the order of discovery determines what these constitutive elements are, the order of discovery is extremely relevant to the justification of a theory (Whewell 1831, 400-401).

To put this another way, although many think that the piecemeal testing of each aspect of a theory is impossible, everyone agrees that it would be better if we could test theories in this manner. On the syntactic view of induction, however, there is no built-in benefit to piecemeal testing; the benefit is external to the logic of confirmation, arising only when it comes time to jettison some part of an unsuccessful theory. For Whewell, by contrast, the benefits of piecemeal testing are built into the logic of confirmation, although, of course, it is not quite a "logic" in a narrow sense. Because a higher-level theory like Newtonian gravity is a semantic induction on the various facts established by other inductions, there is no possible way to verify the fit of this higher-level induction except by confirming its fit with facts established by previous inductions, which means examining each prior induction to determine just what those facts are. For this reason, piecemeal testing is obligatory, and the order of discovery is absolutely essential to the logic of confirmation, as the order determines what aspects of the world our theory must successfully unify in order to survive the test.

\section{Conclusion}

In this article, I have compared Whewell's views on induction to a more familiar contemporary picture - the "syntactic" view of induction I drew from Carnap-and argued that there is a substantial disagreement between them. A natural reaction to this disagreement would be to declare that in our sense of the word, Whewell's "induction" is not induction at all; we can best understand his account of induction by linking it to some other contemporary account of justification, such as hypothetico-deductivism or IBE. What I have tried to show 
in this essay, however, is that the difference between Whewell's account and contemporary views is better understood by comparison with the debates between syntactic and semantic views about theories. Where contemporary accounts generally think of induction as involving logical relations among sentences, Whewell's induction is a type of model-building practice: both the inductive method that he champions and the picture of the resulting scientific knowledge should be understood in terms of constructing a model of the world in the sense of developing an abstract representation. This reading coheres nicely with his broader epistemology. In particular, his views on the consilience and the order of discovery share a natural fit with the semantic picture of induction that they do not have with the more familiar syntactic one.

\section{REFERENCES}

Achinstein, Peter. 1992. "Inference to the Best Explanation; or, Who Won the Mill-Whewell Debate?" Studies in History and Philosophy of Science 23:349-64.

- 2010. "The War on Induction: Whewell Takes On Newton and Mill (Norton Takes On Everyone).” Philosophv of Science 77:728-39.

Buchdahl, Gerd. 1991. "Deductivist versus Inductivist Approaches in the Philosophy of Science as Illustrated by Some Controversies between Whewell and Mill." In William Whewell: A Composite Portrait, ed. Menachem Fisch and Simon Schaffer, 311-44. Oxford: Oxford University Press.

Butts, Robert E. 1967. "Professor Marcucci on Whewell's Idealism.” Philosophy of Science 34:175-83.

Carnap, Rudolf. 1962. Logical Foundations of Probability. Chicago: University of Chicago Press.

Cobb, Aaron D. 2011. "History and Scientific Practice in the Construction of an Adequate Philosophy of Science: Revisiting a Whewell/Mill Debate." Studies in Historv and Philosophy of Science 42:85-93.

Fisch, Menachem. 1991. William Whewell: Philosopher of Science. Oxford: Oxford University Press.

Forster, Malcolm. 2011. "The Debate between Whewell and Mill on the Nature of Scientific Induction." In Handbook of the History of Logic, vol. 10: Inductive Logic, ed. Dov M. Gabbay, Stephan Hartmann, and John Woods, 93-115. Oxford: North-Holland.

Goodman, Nelson. 1979. Fact, Fiction, and Forecast. 4th ed. Cambridge, MA: Harvard University Press.

Harman, Gilbert. 1965. "The Inference to the Best Explanation." Philosophical Review 74:88-95.

Harman, P. M. 1998. The Natural Philosophy of James Clerk Maxwell. Cambridge: Cambridge University Press.

Herschel, John. 1830/1966. Preliminary Discourse on the Study of Natural Philosophy. Repr. New York: Johnson. 
Kant, Immanuel. 1786/2004. Metaphysical Foundations of Natural Science. Trans. and ed. Michael Friedman. Cambridge: Cambridge University Press. Originally published as Metaphysische Anfangsgründe der Naturwissenschaft (Riga: Hartknoch).

Kyburg, Henry. 1990. Science and Reason. Oxford: Oxford University Press.

Mill, John Stuart. 1868/2012. A System of Logic, Ratiocinative and Inductive: Being a Connected View of the Principles of Evidence and the Methods of Scientific Investigation. 7th ed. Repr. Oxford: Benediction Classics.

Myrvold, Wayne. 2003. "A Bayesian Account of the Virtue of Unification." Philosophy of Science 70:399-423.

Norton, John. 2003. "A Material Theory of Induction." Philosophy of Science 70:647-70.

Reid, Thomas. 1785/1941. Essays on the Intellectual Powers of Man. Ed. A. D. Woozley. London: Macmillan. Originally published Edinburgh: Bell.

Snyder, Laura. 2005. "Consilience, Confirmation, and Realism.” In Scientific Evidence: Philosophical Theories and Applications, ed. Peter Achinstein, 129-48. Baltimore: Johns Hopkins University Press.

- 2006. Reforming Philosophy: A Victorian Debate on Science and Society. Chicago: University of Chicago Press.

- 2008. "The Whole Box of Tools': William Whewell and the Logic of Induction." In Handbook of the History of Logic, vol. 4: British Logic in the Nineteenth Century, ed. Dov M. Gabbay and John Woods, 163-228. Oxford: North-Holland.

Thomson-Jones, Martin. 2006. "Models and the Semantic View." Philosophy of Science 73: 524-35.

Whewell, William. 1831. Review of A Preliminary Discourse on the Study of Natural Philosophy, by J. F. W. Herschel. Quarterly Review 45:374-407.

1840/2014a. The Philosophy of the Inductive Sciences, Founded upon Their History. Vol. 1. Repr. Cambridge: Cambridge University Press.

1840/2014b. The Philosophy of the Inductive Sciences, Founded upon Their History.

Vol. 2. Repr. Cambridge: Cambridge University Press.

1849. Of Induction, with Especial Reference to Mr. J. Stuart Mill's System of Logic.

London: Parker. 